\title{
Identifying student conceptual resources for understanding electric current
}

\author{
Lauren C. Bauman \\ Quest University, 3200 University Blvd., Squamish, B.C., Canada, V8B 0N \\ Jonathan Corcoran \\ Department of Education, Seattle Pacific University, 3307 Third Ave W, Seattle, WA, USA, 98119-1997 \\ Lisa M. Goodhew \\ Department of Physics, University of Washington, Box 351560, Seattle, WA, USA, 98195-15603 \\ Amy D. Robertson \\ Department of Physics, Seattle Pacific University, 3307 Third Ave W, Seattle, WA, USA, 98119-1997
}

Most research focusing on student ideas about electric current frames these ideas as misconceptions, difficulties, and misunderstandings. This paper reports student resources for understanding electric current: ideas that we consider to be the "beginnings" of a scientific understanding. Here, we present three conceptual resources for understanding electric current based on our analysis of 550 written responses to conceptual circuits questions from introductory-level university physics students. Our aim is to offer knowledge that can support physics instructors in identifying and building on these resources during instruction. 


\section{INTRODUCTION}

According to Shulman, the specialized knowledge that is needed for teaching (pedagogical content knowledge or PCK) includes both knowledge of content - what is being taught - and knowledge of student ideas (KSI) - the ideas students have about what is being taught [1]. Education researchers have supported the development of instructors' PCK by identifying some of the specific ideas that students bring to bear as they are learning. Previous physics education research informing KSI has largely identified misconceptions, incorrect or unhelpful ideas that cause difficulty and may prevent students from answering questions correctly $[2,3]$. Research on student understanding of topics related to electric current follows this trend; the literature reports a number of difficulties that stem from students' use of incomplete or incorrect ways of thinking about current. For example, students: conceptualize current as dissipating or as "used up" by light bulbs [4-6]; treat batteries as constant sources of current $[4,6,7,8]$; reason locally about current without recognizing the impact of "downstream" elements on the current in the circuit $[4,6,9$, 10]; and do not seem to recognize the need for a complete circuit in order for current to flow [11].

These contributions to KSI highlight reasoning that is discontinuous with formal physics understanding and suggest that this reasoning impedes students' learning of canonically correct physics $[12,13]$. Our work contributes to KSI by identifying students' conceptual resources, useful elements of reasoning that we see as continuous with formal physics [14-16]. Instruction that builds on students' own resourceful thinking has the potential to support students' conceptual understanding, engagement in rich disciplinary practice, and problem-solving processes [17, 18].

This paper presents three common resources for understanding electric current that we identified through analysis of students' written responses to three conceptual questions: (A) Brightness tells us something about current; (B) Breaking or adding a path can change the flow of current in a circuit; and (C) Current that goes in must come out. First, we present our theoretical framework and methods used to arrive at these resources. Then, we discuss examples that illustrate these resources and a breadth of ways they were used in responses. In presenting our analysis, we hope to offer instructors insight into some of the fruitful ideas that students use as they talk about current and how these ideas might be leveraged in introductory physics instruction.

\section{THEORY}

Resources theory conceptualizes thinking as in-themoment activation of pieces of knowledge (i.e., "resources") to construct explanations, arguments, and concepts $[15,16,19]$. In the literature, resources are described as ideas of varying size and internal structure, though they are typically of smaller grain size than scientific concepts. Activation of a particular resource is context-sensitive, being influenced by the learner themselves, the environment, and the question at hand [20]. Resources are not necessarily correct, and can be recognized as sensible and potentially fruitful even when they are activated in contexts where they are incorrect $[15,21]$.

Resources are derived from students' prior experience and learning, and they are sensible; students have good reason for using them as they do [16,19]. Resources theory proposes that learning involves refining, limiting or extending the context for activation of, or strengthening networks of connection between resources [16,20].

In this work, we take up resources theory by analyzing students' responses to written questions about electric circuits for ideas about current that are sensible and potentially fruitful for scientific thinking. Our aim is to provide instructors and researchers with a sense of the resources they may expect introductory physics to use. Thus, we report common, prevalent resources - those that come up in response to multiple questions, for many students.

\section{RESEARCH METHODS}

The resources we report in this paper were identified in written student responses to three circuits questions - the rank-the-bulbs explain question, the compare bulbs series question, and the add-a-wire explain question-all shown in Figure 1. All three were based on questions that were reported in previous literature $[4,5]$.

There are a number of different correct ways to answer the rank-the-bulbs explain and add-a-wire explain questions. One way to do so is to trace the current through the circuit. In the rank-the-bulbs explain question, one can imagine the current traveling through the wires connected to the battery and splitting at the junction. Because bulbs $\mathrm{B}$ and $\mathrm{C}$ are identical, the current splits equally between the two paths, and $\mathrm{B}$ and $\mathrm{C}$ are equally bright. They are less bright than $\mathrm{A}$ and $\mathrm{D}$, which are themselves equally bright, because $\mathrm{A}$ and $\mathrm{D}$ receive all the current from the battery. Adding a wire in the add-a-wire explain question does not change the fact that the current must split between bulbs $\mathrm{A}$ and $\mathrm{B}$; the connections in the circuit do not allow otherwise. The correct answer to the compare bulbs series question is that the bulb in circuit 1 is brighter than the bulb in circuit 2 . Since the resistance of the two (identical) bulbs is the same, and the voltage in circuit 1 is higher than that in circuit 2 because there are two batteries, the current in circuit 2 will be higher by Ohm's law (V = IR).

We analyzed a total of 550 written responses from introductory physics courses at four US colleges and universities: Baylor University, George Mason University (GMU), Howard Community College, and the University of Washington (UW), (Sample size by question and 


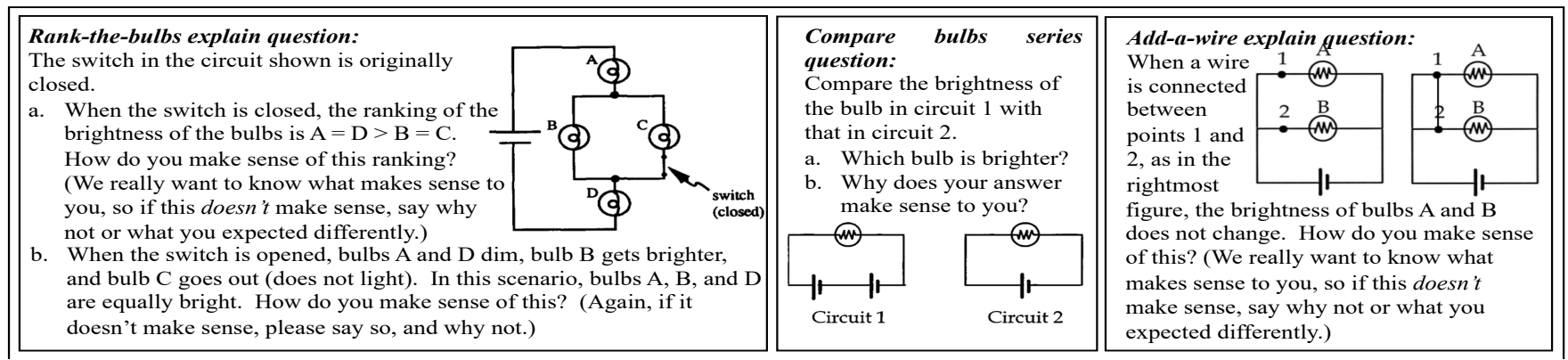

FIG. 1. Three circuits questions used in this study. Figure and parts of text in the rank-the-bulbs explain question reproduced from L. C. McDermott and P. S. Shaffer, "Research as a guide for curriculum development: An example from introductory electricity. Part I: Investigation of student understanding, "American Journal of Physics 60(11), 994, with the permission of the American Association of Physics Teachers. Figures and parts of text from compare bulbs series and add-a-wire explain questions reproduced from P. V. Engelhardt and R. J. Beichner, "Students' understanding of direct current resistive electrical circuits," American Journal of Physics 72(1), 98, with the permission of the American Association of Physics Teachers.

college/university are given in Table I.) Students answered the questions in a variety of contexts: on homework, exams, and quizzes, both pre-and post-instruction. Course response rates were between $90.2 \%$ and $100 \%$ for questions asked at Baylor; $45.8 \%$ for GMU; between $64.8 \%$ and $70.3 \%$ for Howard CC; and $74.7 \%$ for UW.

The racial and/or ethnic demographics for the colleges/ universities in our study versus all college/university students are shown in Fig. 2. Fig. 2 suggests that our study is oversampling from colleges/universities that serve Asian (including Asian American) students and undersampling from colleges/universities that serve Hispanic/Latinx and Black students. In addition, the median parental income of the students at colleges/universities in our study is higher than the national average (calculated from [22]). This overand under-sampling limits the generalizability of our results, and it contributes to a broader trend identified by Kanim and Cid [23], who highlighted that PER in the US has historically oversampled from a small slice of the population of collegebound students (in their meta-analysis, white, wealthy, mathematically-prepared populations of students). This oversampling has both directed the benefits of research and curriculum development to students in these groups and has constructed such students as an implicit norm, reinforcing deficit models of students who are not white, wealthy, and mathematically prepared. One limitation of Figure 2 and our comparison of wealth demographics is that they are based on university-level data, rather than data at the level of our sample. We are accumulating sample-level data, but until we know more about what constitutes a representative sample of introductory physics students, sample-level data does not allow for comparisons to a national average.

We used responses to the three questions in Fig. 1 to create an emergent coding scheme [24] that included some of the resources that the students in our sample were using to reason about circuits. To construct this scheme, authors $\mathrm{LB}$ and $\mathrm{JC}$, in consultation with $\mathrm{AR}$ and $\mathrm{LC}$, conducted preliminary analyses of student responses to each question, looking for ideas that we considered to be continuous with relevant (circuits) formal physics concepts, even if not stated in formal terms. We used these preliminary analyses to identify patterns in student reasoning across questions, foregrounding a model of generalizability that emphasizes recurrence across multiple sources of heterogeneity [25]. This process produced a final scheme with three resources.

Authors LB and JC independently coded student responses to the three questions. Resources can be-and often are-activated in concert; thus, a single response could receive no code, one code, or many codes. As a measure of inter-rater agreement, we took the normalized difference between the total number of possible codes and the total number of disagreements between the two coders. We used percentage agreement rather than a standard statistical measure of agreement (e.g., Cohen's kappa) because our codes are not independent or mutually exclusive [27, 28]. The percentage agreement for the full data set was $86.2 \%$.

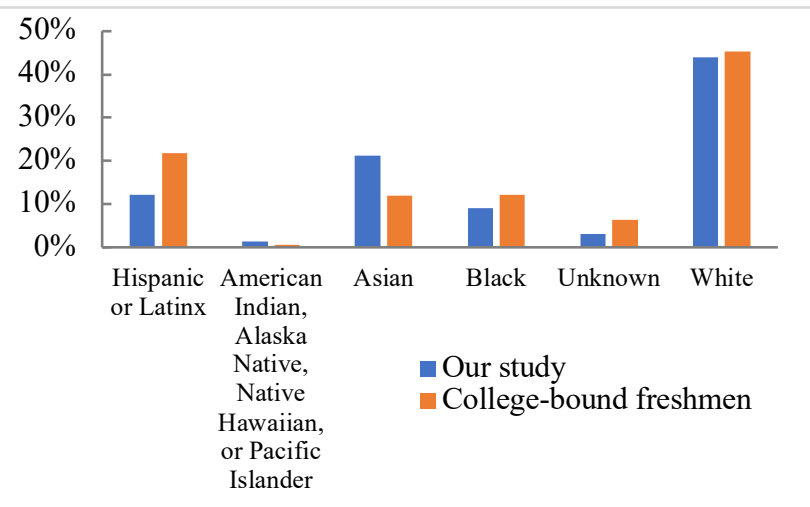

FIG. 2. Racial and/or ethnic demographics of institutions in our sample (blue) versus all college-bound freshmen (orange). Blue bars were constructed using demographic data provided by offices of institutional research or institutional websites, weighted by sample size. Orange bars were constructed using data from Kanim and Cid [23]. As explained by activist Kat Lazo [29], neither Hispanic nor Latinx are racial groups, and these two identities are not the same. "Hispanic" is a descriptor for people of Spanishspeaking origins, and "Latinx" is a descriptor for people with origins in Latin America. The former focuses on language, the latter on geographic location 


\section{RESULTS}

In this section we discuss three common resources for understanding current that we identified from students' responses to circuits questions (Fig.1). Table I presents the relative frequency of each resource for each question and sample.

Importantly, the names of the three resources reflect our best attempt to capture the essence of what students were saying. This essence may not reflect formal physics language, even if it is continuous with formal physics understandings.

TABLE I. Prevalence of each resource by university. Percentages represent responses for which both coders agreed.

\begin{tabular}{rccccccccc}
\hline \hline \multicolumn{10}{c}{ Prevalence } \\
& & Q 1 & & Q 2 & \multicolumn{3}{c}{ Q 3 } \\
& UW & BU & HCC & UW & GMU & UW & GMU & HCC \\
$n$ & 127 & 55 & 24 & 127 & 19 & 127 & 31 & 26 \\
\hline \hline Resource A & $57 \%$ & $58 \%$ & $67 \%$ & $60 \%$ & $38 \%$ & $37 \%$ & $42 \%$ & $23 \%$ \\
Resource B & $48 \%$ & $63 \%$ & $91 \%$ & $1 \%$ & $0 \%$ & $6 \%$ & $10 \%$ & $23 \%$ \\
Resource C & $46 \%$ & $20 \%$ & $46 \%$ & $19 \%$ & $0 \%$ & $11 \%$ & $10 \%$ & $4 \%$ \\
\hline \hline
\end{tabular}

\section{A. Brightness tells us something about current.}

As reflected in Table I, between $23 \%$ and $67 \%$ of the student responses in our samples used the idea that brightness is a visual indicator of current. These responses reflect an association between a model-based quantity (current) and a concrete observation (brightness). That current is associated with brightness means both (i) that brightness can indicate current - i.e., that students can use an observation of the brightness of bulbs to say something about what is happening in the wires - and (ii) that one can use current to predict brightness-i.e., that students can make predictions about the brightness of bulbs based on a conceptual or mathematical understanding of current. We assigned this code in both directions.

For example, one student used current-based reasoning to justify the equal brightness of bulbs $\mathrm{A}$ and $\mathrm{D}$ in the rank-thebulbs explain question (Fig.1): "The brightness is determined by the current flowing through the bulbs. The current at $\mathrm{A}$ equals the current at $\mathrm{D}$, as they are both the only paths for current to flow within their sections of the circuit. The current at $\mathrm{B}$ equals the current at $\mathrm{C}$, as they are both one of two possible paths for the current to flow within their section of the circuit. If the current at $\mathrm{A}$ is 2 , at $\mathrm{B}$ and $\mathrm{C}$ it will be 1 , and at $\mathrm{D}$ it will be 2 again." This student identifies a relationship between current flow and brightness as they trace the path of current through the wires, mapping the amount of current in different parts of the wires to brightness. Other responses drew on the relationship between current, voltage, and resistance to justify the relative brightness of bulbs. For example, in response to the compare bulbs series question, a student stated: "Brightness can be looked at as 'equivalent' to current [...] so if current $=\mathrm{V} / \mathrm{R}$ and circuit 1 has $2 \mathrm{~V} / \mathrm{R}$, circuit 2 has $\mathrm{V} / \mathrm{R}$, then I [current] is greater on circuit 1 , meaning that the bulb on circuit 1 will glow brighter." Instead of tracing current, this student justifies their response using Ohm's Law, explaining there is more current in circuit 1 because there is more voltage. In both of these examples, students are explaining differences in brightness (observation) in terms of a difference in current (model).

\section{B. Breaking or adding a path can change the flow of current in a circuit.}

Anywhere from 0 to $91 \%$ of student responses in our samples drew on the idea that breaking or adding a path can change the flow of current in a circuit. We specifically applied this code to student responses that identified how opening or closing switches or adding extra paths would result in a change in the amount of current in different parts of the circuit. For example, one student justified their response to the rank-the-bulbs explain question by saying, "Bulb B gets brighter because instead of current being split at the junction, it will just flow through bulb B because current won't flow through [bulb] $\mathrm{C}$ because it is incomplete." This response highlights that current will flow through both paths when the path through bulb $\mathrm{C}$ is complete but will not flow through bulb $\mathrm{C}$ when the path through it is broken.

This resource was most frequently used in the rank-thebulbs explain question, to justify why bulb $\mathrm{C}$ does not light. However, we also observed instances of similar reasoning in Q3 (add-a-wire explain question). For example, a student wrote, "Because a current from the battery through the wire from 1 to 2 will still have to pass through either bulb A or B to complete the loop." Here, the student justifies that there will be no change in brightness because they cannot find a path where the current would not flow through each bulb.

Reasoning like that used in these examples - and in other examples that received this resource code-is continuous with important aspects of canonical understandings of potential difference and conservation of current. In particular, the notion that there must be a complete loop connecting a circuit element back to a voltage source in order for current to flow enforces current conservation-the current cannot just disappear or dissipate - and points to the need for a potential difference in order for current to flow.

\section{Current that goes in must come out.}

The resource that "current that goes in must come out"identified in $0 \%$ to $46 \%$ of student responses in our samples - instantiates a more overt notion of charge or current conservation, and in some cases may represent an intuitive version of Kirchhoff's junction rule. Responses that used this resource emphasized that when current splits at a junction, the same amount of current must exit the junction, 
and/or that the amount of current that leaves a battery must return to the battery.

Sometimes, students expressed these ideas by using language like "splitting" or "sharing" current. For example, in response to the rank-the-bulbs explain question, a student wrote, "...Current splits and then recombines. Therefore, A and D would receive full current and B and $\mathrm{C}$ would share that same current because it splits." Other students expressed these ideas quantitatively, accounting for the current in different parts of the circuit as a fraction of the total current. For example, a student reasoned, " $\mathrm{A}=\mathrm{D}$ because both get same current. $B=C$ because same current but $1 / 2$ of the original $(\mathrm{A}=\mathrm{D})$. So, $\mathrm{A}=\mathrm{D}>\mathrm{B}=\mathrm{C}$." Instead of explicitly describing the current as separating at a junction and "recombining" later, this student assigns half the total current to the bulbs on each branch. Finally, this resource was used in responses that explicitly stated that current is conserved, or that used Kirchoff's loop law. For example, one student used Kirchoff's loop law to explain why bulb 1 is brighter than bulb 2 in the compare bulbs series question, writing: "In circuit 1 the loop rule is $V_{1}+V_{2}-I_{1} R_{1}=0$, [and so] $I_{1}=\frac{2 V}{R}$ and in circuit 2 the loop rule is $V_{1}-I_{2} R_{2}=$ 0 , [and so] $I_{2}=\frac{V}{R}$ so $I_{1}>I_{2}$ and bulb 1 will be brighter."

\section{DISCUSSION}

This paper presents three conceptual resources for understanding current that were common in our sample: (A) Brightness tells us something about current; (B) Breaking or adding a path can change the flow of current in a circuit; and (C) Current that goes in must come out. We identified these resources through analysis of 550 written responses to conceptual circuits questions, seeking elements of thinking that are continuous with formal physics, even if not fully correct (or correct in all contexts). The generalizability of our results is limited by the nonrepresentativeness of our sample (Fig. 2); the extent to and ways in which population variability will change results like these is an open question [23].

In identifying and communicating these resources, our aim is to support the development of instructors' PCK by adding to existing, difficulties- or misconceptions-oriented research about students' understanding of electric circuits. In particular, prior literature suggests that students encounter a number of difficulties with electric circuits, and that many of these are rooted in misunderstandings of electric current. For example, students think of current as used up; reason locally about current (e.g., think the order of elements matters); or do not recognize the need for a complete circuit in order for current to flow [4-11]. Our research offers a different story. In our analysis, students reason in ways consistent with current conservation (Resources B and C); they use indicators of current that support their predictions about bulbs in series and parallel (Resource A); and they identify the effects of breaking a complete circuit (Resource B). Whether this story is different because students are reasoning differently in the questions we asked (when and how we asked them) or because we brought a different lens to the data is not clear. What is clear is that students have many useful ideas about current that could serve as starting points for instruction-ideas which instructors can activate, leverage, and build on towards deeper conceptual understanding.

For example, responses using Resource A explain or predict physical observations such as a light bulb dimming or brightening using a physical model for what is happening in the wire. Resources B and C imply or explicitly point to current conservation. Instructors could listen for these and other productive ideas about current, leveraging and refining them alongside students to deepen conceptual understanding. For instance, an instructor may wish to build upon or refine students" notion of "splitting" and "rejoining" (Resource C) by emphasizing how this idea aligns with a fundamental law of conservation, or by inviting students to formulate a mathematical representation of their ideas.

Instructors may also attempt to elicit particular resources using our questions or their own. Though this work is still preliminary, Table I both affirms resource theory's prediction that resources are context-dependent $[19,20]$ and suggests that some contexts may more reliably elicit a given resource than others. For example, Resource B is elicited at consistently substantive (though variable) frequencies in the rank-the-bulbs explain question and at consistently small frequencies in the compare bulbs series question. (This makes sense, because a salient feature of the rank-the-bulbs question is opening a switch.) Future research may provide more clarity about resource-question pairings.

\section{ACKNOWLEDGMENTS}

This work was supported in part by the National Science Foundation under grant numbers $1914603 \& 1914572$. The authors wish to thank Paula R. L. Heron, Rachel E. Scherr, Raphael Mondesir, Brynna Hansen, Cheyenne Broadfoot for offering helpful feedback, and all those who helped with data collection. 
[1] L. Shulman, Harv. Educ. Rev. 57, 1 (1987).

[2] L. C. McDermott and E. F. Redish, Am. J. Phys. 67, 9 (1999).

[3] D. E. Brown and D. Hammer, in International Handbook of Research on Conceptual Change, edited by S. Vosniadou (Routledge, New York, NY, 2008), p. 127-154.

[4] L. C McDermott and P.S. Shaffer, Am. J. Phys. 60, 994-1003 (1992).

[5] P. V. Engelhardt and R.J. Beichner, Am. J. Phys. 72, 98-115 (2003).

[6] D. Shipstone, Phys. Ed. 23, 92 (1988).

[7] D. P. Smith and P. van Kampen, Phys. Rev. ST Phys. Educ. Res. 7, 020115 (2011).

[8] R. Cohen, B. Eylon and U. Ganiel, Am. J. Phys. 51, 407-12 (1983).

[9] D. M. Shipstone, Eur. J. Sci. Ed. 6, 185-98 (1984).

[10] V. Picciarelli, M. D. Gennaro, R. Stella and E. Conte, Eur. J. Eng. Ed. 16, 41-56 (1991).

[11] M. R. Stetzer, P. van Kampen, P.S . Shaffer and L. C. McDermott, Am. J. Phys. 81, 134-43 (2013).

[12] L. C. McDermott, in Toward a Scientific Practice of Science Education, edited by M. Gardner, J. G. Greeno, F. Reif, A. H. Schoenfeld, A. diSessa, \& E. Stage (Lawrence Erlbaum Associates, Hillsdale, NJ, 1990).

[13] G. J. Posner, K. A. Strike, P. W. Hewson and W. A. Gertzog, Sci. Ed. 66, 2 (1982).

[14] D. Hammer and E. van Zee, Seeing the Science in Children's Thinking: Case Studies of Student Inquiry in Physical Science (Heinemann, Portsmouth, NH, 2006).

[15] D. Hammer, Am. J. Phys. 69, S52 (2000).

[16] J.P. Smith, A.A. diSessa, and J. Roschelle, J. Learn. Sci. 3, 2 (1993).

[17] D. Hammer, F. Goldberg and S. Fargason, Rev. Sci. Math, and ICT Ed. 6, 1 (2012).

[18] D. Hammer, Cognition Instruct. 15, 4 (1997).

[19] A. A. diSessa, Cognition Instruct. 10, 2 \& 3 (1993).

[20] D. Hammer, A. Elby, R. E. Scherr, and E. F. Redish, in Transfer of Learning from a Modern

Multidisciplinary Perspective, edited by J.P. Mestre (Information Age Publishing, Greenwich, 2005).

[21] R. E. Scherr, Am. J. Phys. 75, 272 (2007).

[22] http://www.equality-of-opportunity.org/data/. Retrieved 4/29/2020.

[23] S. Kanim and X.C. Cid, Phys. Rev. Phys. Educ. Res. 16, 2 (2020).

[24] K. Krippendorff, Content Analysis: An Introduction to Its Methodology 3rd ed. (Sage, Thousand Oaks, 2013)

[25] T.D. Cook, Educ. Eval. Policy An. 24, 3 (2002).

[27] M. L. McHugh, Biochem. Medica, 22, 276 (2012).

[28] J. Cohen, Educ. Psychol. Meas., 20, 37 (1960).

[29] https://www.vox.com/2016/8/28/12658908/latinohispanic-race-ethnicity-explained. Retrieved 5/19/2020. 